\title{
Anxiolytic-like activity of leaf extract of traditionally used Indian-Mustard (Brassica juncea) in diabetic rats
}

\author{
Ajit Kumar Thakur ${ }^{1}$, Shyam Sunder Chatterjee ${ }^{2, \dagger}$, Vikas Kumar $^{1, *}$ \\ ${ }^{I}$ Neuropharmacology Research Laboratory, Department of Pharmaceutical Engineering, Indian Institute of Technology (Banaras \\ Hindu University), Varanasi-221 005, India; ${ }^{2}$ Stettiner Straße 1, D-76138 Karlsruhe, Germany
}

\begin{abstract}
Brassica juncea is a polyphenols enriched edible plant, with diverse medicinal uses of different parts of which have been mentioned in the Ayurveda. The effects of 10 daily oral doses (100, 200, and 400 $\mathrm{mg} / \mathrm{kg} /$ day) of a methanolic Brassica juncea leaf extract in rat models of anxiety using nondiabetic and alloxan-diabetic rats were quantified. In all the three behavioural tests used, i.e. elevated plus maze, open field, and social interaction tests, anxiolytic-like activity of the extract was observed in the diabetic animals only. Quantitatively, the efficacy of the highest tested dose of the extract in these tests was always less than those observed after its lower ones. These observations provide further experimental evidences for the conviction that Brassica vegetables could as well be useful for combating diabetes associated mental health problems.
\end{abstract}

Keywords Brassica juncea, anxiety, comorbidities, diabetes mellitus

\section{INTRODUCTION}

Diabetes mellitus is a spreading epidemic of the $21^{\text {st }}$ century, and India has a higher prevalence of diabetes than any other nation (Diamond, 2011). Herbal remedies continue to be to be major therapeutic options of diabetic patients in this country (Malvi et al., 2011), and extensive efforts to properly understand and define their therapy relevant pharmacological activity profiles have lead to a long list of edible and other plants with antidiabetic activity in animal models (Mukherjee et al., 2006). Little attention is given to the fact that the progression of diabetes leads to diverse mental health problems which are major obstacles for proper glycaemic control for avoiding these diabetes associated comorbidities (Herzer and Hood, 2010). Diabetes associated comorbid psychopathologies are the major causes of health care costs of diabetes (Hutter et al., 2010) and available psychotherapeutics do not appropriately meet the therapeutic demands of diabetic patients. Moreover, many currently available psycho active drugs possess obesogenic potentials, and obesity is a major risk factor of insulin resistance and type 2 diabetes (Faulkner et al., 2009; Pan et al., 2012).

It is now well recognized that diabetes and psychiatric disorders share a bidirectional association (Balhara, 2011), and that the gut-central nervous system could as well be the target for nutritional therapies (Pimentel et al., 2012). The crucial role of gut microbiota in the etiology, pathogenesis and progression of diabetes and associated comorbidities is now becoming increasingly apparent (Sekirov et al., 2010) and bacteriostatic activities of numerous phytochemical consumed with food and

"Correspondence: Vikas Kumar

E-mail: vikas.phe@iitbhu.ac.in

'Retired Head of Pharmacology Research Laboratories, Dr. Willmar Schwabe GmbH \& Co. KG., Karlsruhe, Germany

Received November 17, 2012; Accepted February 21, 2013; Published February 28, 2013

doi: http://dx.doi.org/10.5667/tang.2012.0042

(C)2013 by Association of Humanitas Medicine

TANG / www.e-tang.org herbal remedies are well known. Therefore, it is possible that traditionally known medicinal uses of plants and spices in the Indian system of medicine could as well be due to their actions on the microbiota-gut-brain axis. Accordingly, attempts to identify edible and other Ayurvedic medicinal plants potentially useful for combating diabetes-associated depression, anxiety, and cognitive abnormalities are now being made in our laboratories. Literature searches made during initiation of the project led us speculate that plants of the mustard family (Brassicaceae) could be a promising starting point for such purposes.

Brassica juncea, also called Indian-mustard, is an economically important edible plant, with diverse medicinal uses of which are well known to practitioners of the Ayurveda and other traditionally known medical systems currently widely practiced in India (Manohar et al., 2009). According to Ayurvedic principles, proper balance between the functions of body and mind is essential for healthy living. Although recent reports have revealed the antihyperglycemic activity of methanolic and other types of Brassica juncea leaf extracts (Manohar et al., 2009; Rahmatullah et al., 2010; Valavala et al., 2011), as yet little attention has been paid to the potential benefits of such extracts on the central nervous system. Our critical analysis of available information on medicinal uses, pharmacological properties, and bioactivities secondary metabolites of the plant led us to the working hypothesis that the methanolic extract of dried Brassica juncea leaves (BJ) could as well be useful for combating diabetes-associated mental health problems (Kumar et al., 2011). Consequently, efforts to compare the psychopharmacological activity profiles of the extract in diabetic and nondiabetic animals were made in our laboratories. The extract contains isorhamnetin and sinapic acid, and these secondary plant metabolites have been reported to possess antidepressant and anxiolytic-like activities respectively (Chatterjee, et al., 2005; Yoon et al., 2007). In a first set of experiments, a methanolic Brassica juncea leaf extract containing these two polyphenols was pharmacologically screened in a battery of animal behavioural 
models commonly used for identifying antidepressants-like activities of bio-active agents. Observations made during these efforts revealed that dose-dependant antidepressant-like activity of the extract can be detected in diabetic animals only (manuscript submitted for publication). Efforts made to detect anxiolytic-like activity of the extract revealed again that the extract can effectively antagonize exaggerated anxiety in diabetic rats, and that it has no effect on the anxiety state of nondiabetic animals. These observations are reported in this communication. Some potential implications of our findings for understanding holistic therapeutic possibilities offered by edible and other plants are also discussed in short.

\section{MATERIALS AND METHODS}

\section{Plant material and extraction}

Brassica juncea leaves were collected from a local agricultural area in Varanasi (U.P., India), and were botanically authenticated by Prof. N. K. Dubey in Herbarium of Department of Botany, Banaras Hindu University as Brassica juncea (Linn.) species Czern \& Coss family Brassicaseae (Voucher specimen number Dubey 12/ Nov/ 2009). They were dried at room temperature and powdered. The leaf extract used in this study was prepared by exhaustive soxhlet extraction of the powder $(800 \mathrm{~g})$ with two liters of aqueous methanol $(90 \%)$ for $3 \mathrm{~h}$. The solvent was evaporated, and the extract was dried in a vacuum at $40^{\circ} \mathrm{C}$. The calculated yield of the extract (BJ) was $11 \%$ by weight of the dried leaves.

\section{Extract characterization}

A well-validated HPLC method described elsewhere (Chandrasekaran et al., 2009) and commonly used for quantifying total flavonoids contents of plant extracts was chosen. Briefly, a Shimadzu LC 2010HT HPLC system equipped with a quaternary pump, UV detector, degasser and an auto sampler with "Lab solution" software was used. Mobile phase was acetonitrile: phosphate buffer ( $\mathrm{pH} 2.5$ - 2.8). Column was C18- ODS (octadecyl silane) $5 \mu$ size, 250 x $4.6 \mathrm{~mm}$, wavelength: $280 \mathrm{~nm}$, flow rate: $1.5 \mathrm{ml} / \mathrm{min}$, injection volume: $20 \mu 1$ and run time was 40 minute. Using a slightly modified spectrophotometric method (Malgorzata and Aleksander, 2010) the total sinapic acid content of the extract was analyzed.

\section{Animals}

Adult Charles foster rats $(160 \pm 20 \mathrm{~g})$ and albino mice $(20 \pm 5 \mathrm{~g})$ were obtained from Central Animal House of Institute of Medical Sciences, Banaras Hindu University, Varanasi. They were housed in groups of six in polypropylene cages at an ambient temperature of $25 \pm 1^{\circ} \mathrm{C}$ and $45-55 \%$ relative humidity, with a 12:12 h light/dark cycle. Except when stated otherwise, they were always provided with commercial food pellets and water ad libitum. All animals were acclimatized to laboratory conditions for at least one week before using them for the experiments. Principles of laboratory animal care (NIH publication number 85 - 23, revised 1985) guidelines were followed, and prior approval for animal experimental work from the Central Animal Ethical Committee of the University was obtained.

\section{Induction of diabetes}

Insulin dependent diabetes mellitus (Type 1-like diabetes, also called "alloxan diabetes") was induced after an overnight fast by a single i.p. injection of alloxan monohydrate $(120 \mathrm{mg} / \mathrm{kg}$ body weight) in normal saline (Lenzen, 2008). One h after the injection of alloxan all animal were provided with commercial food pellets and water ad libitum. Hyperglycemia was confirmed by fasting blood glucose level measurements on the $3^{\text {rd }}$ and $7^{\text {th }}$ days after the alloxan injection. Preselected diabetic animals with hyperglycemia on the $7^{\text {th }}$ day (fasting blood glucose levels $>250 \mathrm{mg} / \mathrm{dl}$ ) were used for further studies.

\section{Animal grouping and drug administration}

Nine groups of six animals each ( 3 males and 3 females in each group) were used for the experiments. Treatments of the diabetic groups started on the $7^{\text {th }}$ day the after alloxan challenge (day 1 of treatment). Suspensions of BJ in $0.3 \%$ carboxymethyl cellulose (CMC) were orally administered once daily for ten consecutive days. The treatment groups were: Group I: Nondiabetic control (CMC treated), Group II: Nondiabetic treated with BJ (100 mg/kg/day); Group III: Nondiabetic treated with BJ (200 mg/kg/day); Group IV: Nondiabetic treated with BJ (400 mg/kg/day); Group V: Diabetic control (CMC treated); Group VI: Diabetic treated with BJ (100 $\mathrm{mg} / \mathrm{kg} /$ day); Group VII: Diabetic treated with BJ (200 $\mathrm{mg} / \mathrm{kg} /$ day); Group VIII: Diabetic treated with BJ (400 $\mathrm{mg} / \mathrm{kg} /$ day); and Group IX: Diabetic treated with a lorazepam (1 mg/kg/day), a well-known anxiolytic drug.

\section{Elevated plus maze test for rats}

The method described by Pellow and File (1986) was followed. The maze had two opposite arms, $50 \times 10 \mathrm{~cm}$, crossed with two enclosed arms of the same dimension but having $40 \mathrm{~cm}$ high walls. The arms were connected with a central square, $10 \times 10$ $\mathrm{cm}$, giving the apparatus the shape of a plus sign. The maze was kept in a dimly lit room and elevated $50 \mathrm{~cm}$ above the floor. Rats were placed individually in the centre of the maze, facing an enclosed arm. Thereafter, the number of entries and time spent on the open and enclosed arms were recorded during the next $5 \mathrm{~min}$ and data expressed as \% entry in open arms and \% time in open arms.

\section{Open field test for rats}

The method described by Bronstein (1972) was followed. The open field apparatus was made of plywood and consisted of squares of $(61 \times 61 \mathrm{~cm})$. The entire apparatus was painted black except for $6 \mathrm{~mm}$ thick white lines, which divided the floor into 16 squares. The open-field was lighted by a $40 \mathrm{w}$ bulb focusing on the field from a height of about $100 \mathrm{~cm}$. The entire room, except the open-field, was kept dark during the experiment. Each animal was centrally placed in the test apparatus for $5 \mathrm{~min}$ and the following four behavioural aspects. Ambulation: this was measured in terms of the number of squares crossed by the animal; Rearing: number of times the animal stood on its hind limbs; Self-grooming: number of times the animal groomed the facial region, and licked/washed/scratched various parts of its body; Activity in the centre: number of central squares crossed by the animal; and Faecal droppings: number of faecal droppings excreted during the period was recorded.

\section{Social interaction test in rats}

The method described by File and Hyde (1978) was followed. Rats were first housed individually for 8 days before testing. The apparatus used for the test was a wooden box $(60 \times 60 \times$ $35 \mathrm{~cm}$ ) with a solid floor and was placed in a dimly lit room. On day 9 of the treatments, the rats were placed individually in the box again, and given two $7.5 \mathrm{~min}$ familiarization sessions at a $2 \mathrm{~h}$ interval. On day 10, another rat was placed simultaneously (paired on weight and sex basis) in the box for 7.5 min. During this time, the total time spent by the rat pair in "social interaction", including sniffing, following, grooming, kicking boxing, biting and crawling under or over the partner, was recorded by a neutral 'blind' observer. 


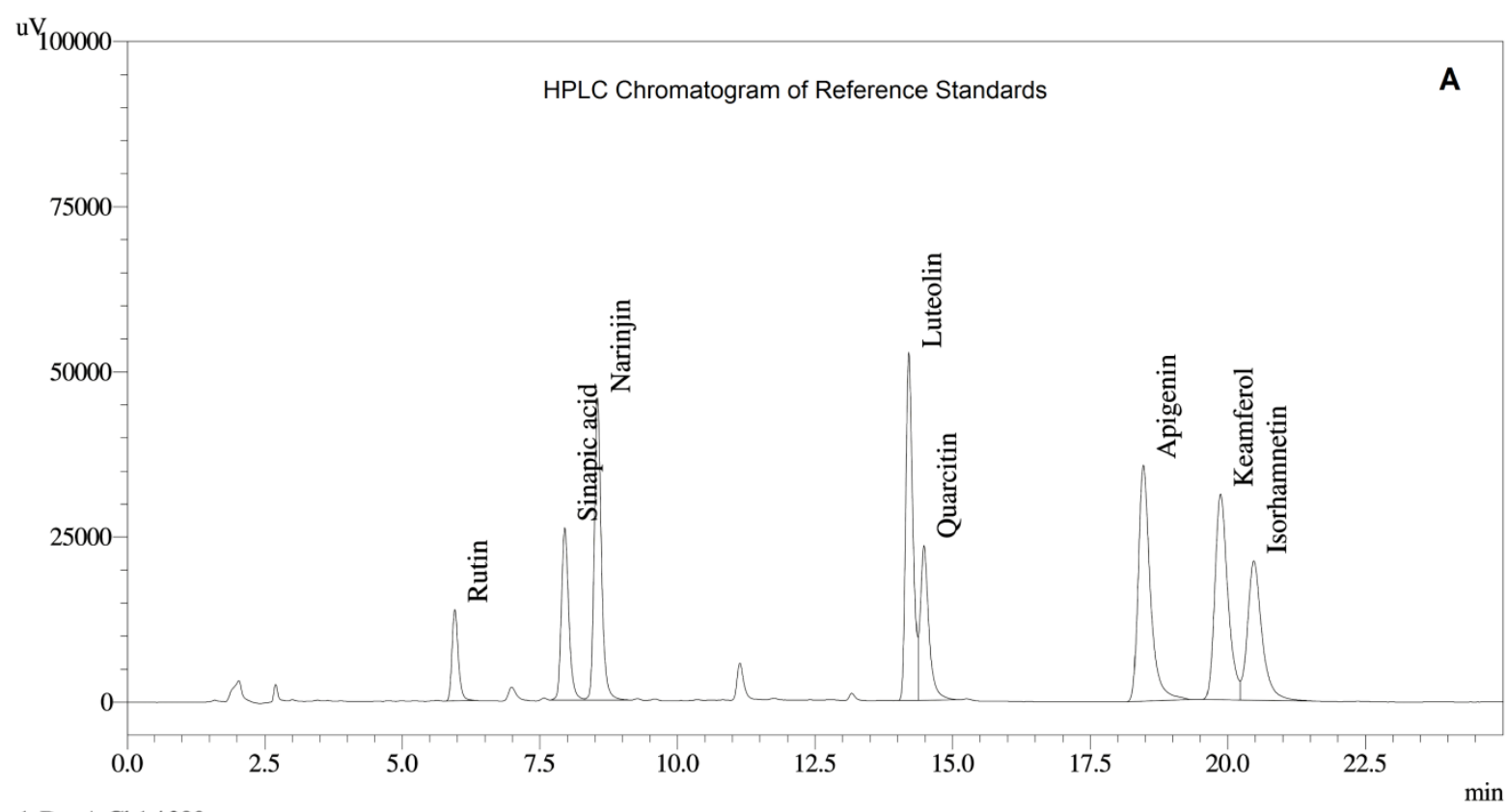

1 Det.A Ch1 / 280nm

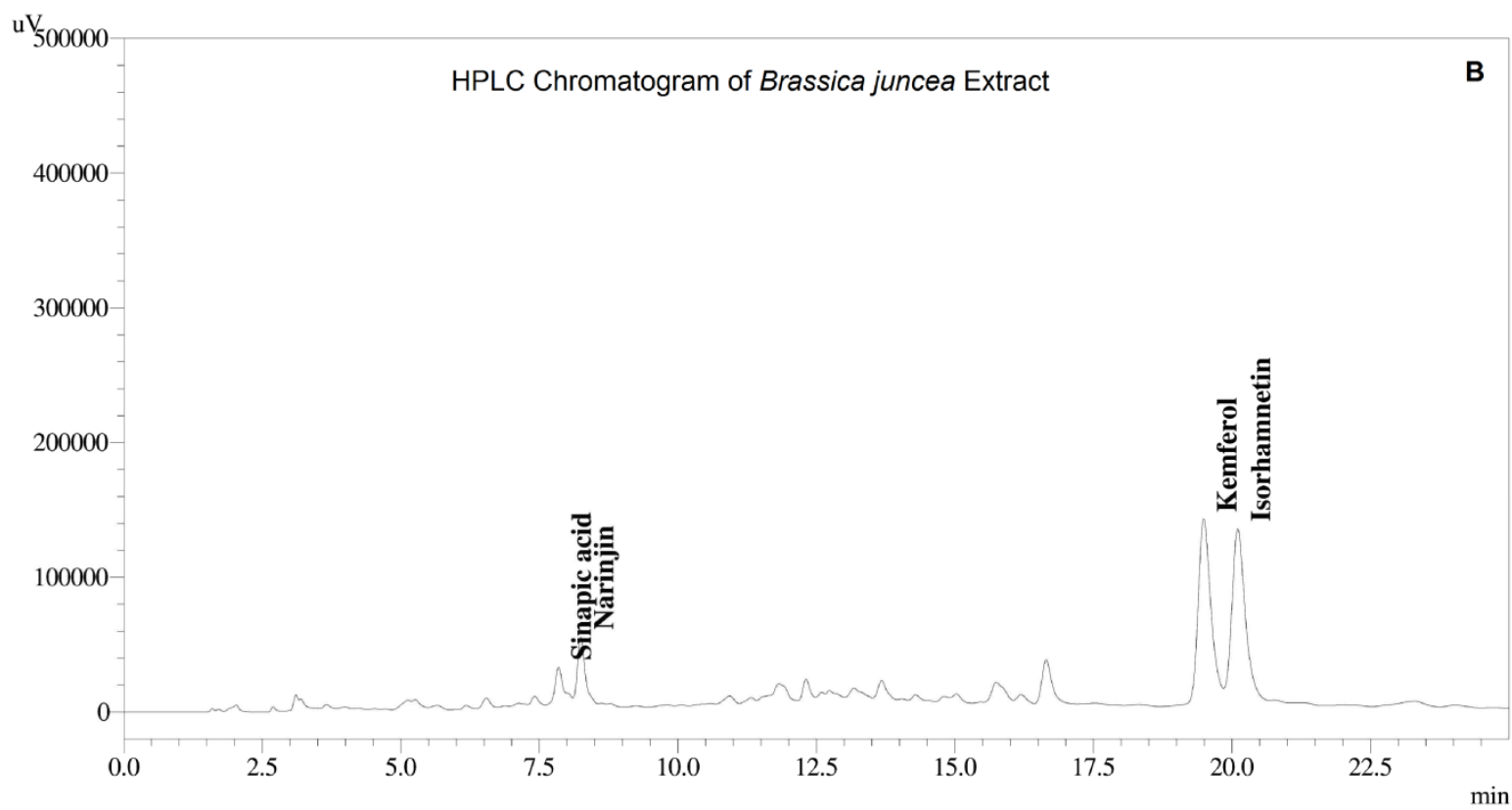

1 Det.A Ch1 / 280nm

Fig. 1. Analytical characterization of Brassica juncea extract. HPLC Chromatogram of (A) polyphenols standard and (B) a hydrolyzed sample of the methanolic Brassica juncea leaf extract.

\section{Statistical analysis}

Mean \pm standard errors of mean $(n=6)$ were calculated for the observed values in each experimental group. Statistical analysis was performed by a one-way analysis of variance (ANOVA) followed by a Student-Newman-Keuls multiple comparison test. GraphPad Prism 5 was used for statistical analysis. A p-value less than 0.05 were considered as statistically significant.

\section{RESULTS}

Extract characterization

HPLC chromatograms as shown in Fig. 1A and 1B revealed that $\mathrm{BJ}$ contains $0.37 \%$ kaemferol and $0.29 \%$ isorhamnetin total flavonoids. The sinapic acid content of the extract was found to be $5 \%$ of total phenolic contents by spectrophotometric method.

\section{Elevated plus maze test}

In nondiabetic rats, BJ treatments did not have any significant effects on the number of entries in open arms or on the time spent in them. As compared to the nondiabetic control group, mean $\%$ entries and $\%$ time spent by the diabetic control group in those arms were significantly decreased. As can be seen from the results summarised, such were not the cases for BJ- and lorazepam-treated diabetic groups (Fig. 2). Surprisingly though, quantitatively, the effects of the highest BJ doses (400 


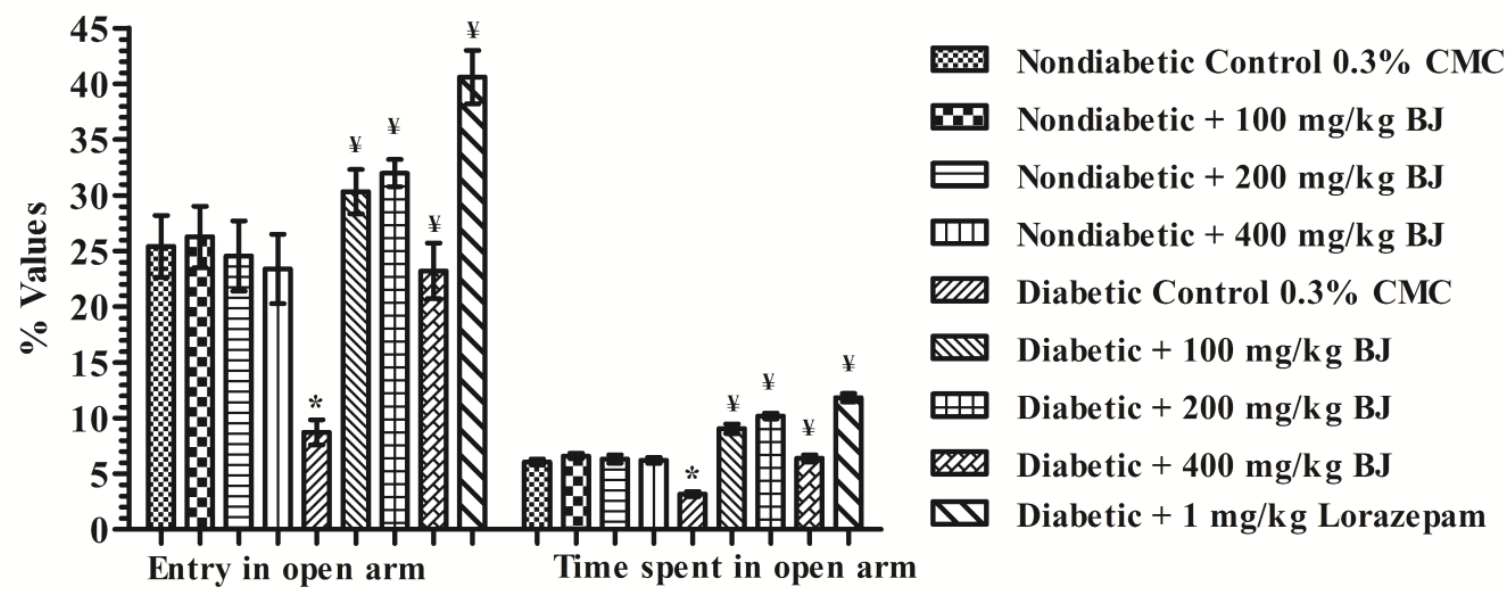

Treatment groups

Fig. 2. Effect of Brassica juncea on elevated plus maze behaviour in nondiabetic and diabetic rat. * $p<0.05$ vs. Nondiabetic Control; ${ }^{¥} p<0.05$ vs. Diabetic Control.

$\mathrm{mg} / \mathrm{kg} /$ day) in diabetic rats were lower than those of the two lower ones tested (100 and $200 \mathrm{mg} / \mathrm{kg} /$ day $)$.

\section{Open field test}

Results of this test are summarized in Table 1. As compared to the corresponding values in the nondiabetic control group, the mean values of ambulation, central activity, rearing and self grooming in the diabetic control group were significantly lower. The mean numbers of fecal droppings in the diabetic control rats were also significantly higher than in the nondiabetic groups. Although BJ treatments did not have any effects on these parameters in nondiabetic rats (results not shown), all mean values of the BJ- or lorazepam-treated diabetic groups were significantly different from those of the CMC-treated diabetic group. Once again, quantitatively, the efficacy of the $400 \mathrm{mg} / \mathrm{kg} / \mathrm{day}$ BJ-treated group was lower than those of the other two groups treated with lower doses of the extract (100 and $200 \mathrm{mg} / \mathrm{kg} / \mathrm{day}$ ). The observed effects of the 100 and 200 $\mathrm{mg} / \mathrm{kg} / \mathrm{day}$ BJ-treated diabetic groups were not significantly different.

\section{Social interaction test}

Mean social interaction times of CMC-treated diabetic group were significantly lower than those of the CMC-treated nondiabetic group (Fig. 3). Although BJ treatments had no significant effects on nondiabetic rats, lorazepam-like anxiolytic effects of the extract were apparent in the diabetic animals. As shown in Fig. 3, quantitatively this observed effect of $400 \mathrm{mg} / \mathrm{kg} / \mathrm{day} \mathrm{BJ}$ was again much lower than those observed after its lower two doses, and that the effects of 100 and $200 \mathrm{mg} / \mathrm{kg} / \mathrm{day}$ BJ treatments were not significantly different in magnitude. These observations indicate again that the dose response curves of $\mathrm{BJ}$ might be inverted $\mathrm{U}$-shaped in the rodent models of anxiety, and reconfirm that the 100 $\mathrm{mg} / \mathrm{kg} / \mathrm{day}$ dose of the extract is high enough for observing its psychopharmacological activities.

\section{DISCUSSION}

Diabetes is a chronic endocrine disorder leading to hyperglycemia and other metabolic abnormalities. Uncontrolled diabetes leads to disabling and life threatening conditions and associated mental health problems. Brassicaceae vegetables represent an important part of the human diet worldwide, and available information on the pharmacological activity profiles of their diverse types of extracts and bio-active components strongly suggest their preventive and therapeutic potentials against diverse spectrum of comorbidities associated with, or caused by environmental challenges (Jahangir et al., 2009). Brassica juncea is a member of this family of plants, the edible leaves of which are often used for culinary purposes in Indian and other countries. Although the antihyperglycemic activity of Brassica juncea leaves (Grover et al., 2002; Rahmatullah et al., 2010; Valavala et al., 2011), and numerous bioactive and extractable secondary plant metabolites encountered in them are known (Kumar et al., 2011), reports on their therapeutically interesting psychopharmacological properties are rare, or scattered. Results of the initial pharmacological screening efforts made in our laboratories revealed that the methanolic Brassica juncea leaf extract (BJ) not only possesses an antihyperglycemic activity in alloxan diabetic animals, but also dose dependently reverses the exaggerated anxious behavioural symptoms observed in such animals. However, no antidepressant-like efficacy of the extract was observed in normal laboratory rodents (Unpublished data). Since these beneficial effects of the extract compensated for also the bodyweight losses accompanying alloxan-induced diabetes, we speculated that its observed effects could as well be due to its insulin sensitizing effects. Analogous suggestions for the mode

Table 1. Effect of Brassica juncea on open field exploratory behaviour in rats

\begin{tabular}{|c|c|c|c|c|c|}
\hline Treatment groups & Ambulation & Central activity & Rearing & Self grooming & Faecal dropping \\
\hline Nondiabetic Control (0.3\% CMC) & $54.33 \pm 0.95$ & $2.83 \pm 0.30$ & $9.33 \pm 0.33$ & $5.00 \pm 0.25$ & $7.16 \pm 0.30$ \\
\hline Diabetic Control (0.3\% CMC) & $38.83 \pm 0.94 *$ & $1.16 \pm 0.16^{*}$ & $5.50 \pm 0.34^{*}$ & $2.66 \pm 0.21^{*}$ & $9.66 \pm 0.33^{*}$ \\
\hline Diabetic $+100 \mathrm{mg} / \mathrm{kg} \mathrm{BJ}$ & $60.83 \pm 0.74^{\ddagger}$ & $3.33 \pm 0.33^{¥}$ & $13.16 \pm 0.60^{¥}$ & $4.50 \pm 0.42^{¥}$ & $7.33 \pm 0.33^{¥}$ \\
\hline Diabetic $+200 \mathrm{mg} / \mathrm{kg} \mathrm{BJ}$ & $65.48 \pm 0.91^{¥}$ & $3.86 \pm 0.56^{¥}$ & $14.89 \pm 0.49^{¥}$ & $5.12 \pm 0.75^{¥}$ & $6.89 \pm 0.81^{¥}$ \\
\hline Diabetic $+400 \mathrm{mg} / \mathrm{kg} \mathrm{BJ}$ & $46.00 \pm 1.63^{¥}$ & $2.83 \pm 0.30^{¥}$ & $8.00 \pm 0.36^{¥}$ & $4.83 \pm 0.30^{¥}$ & $8.00 \pm 0.36^{¥}$ \\
\hline Diabetic $+1 \mathrm{mg} / \mathrm{kg}$ Lorazepam & $70.66 \pm 1.70^{¥}$ & $4.83 \pm 0.30^{¥}$ & $16.16 \pm 0.60^{¥}$ & $7.16 \pm 0.47^{¥}$ & $6.16 \pm 0.30^{\ddagger}$ \\
\hline
\end{tabular}

$* p<0.05$ vs. Nondiabetic Control; ${ }^{*} p<0.05$ vs. Diabetic Control 


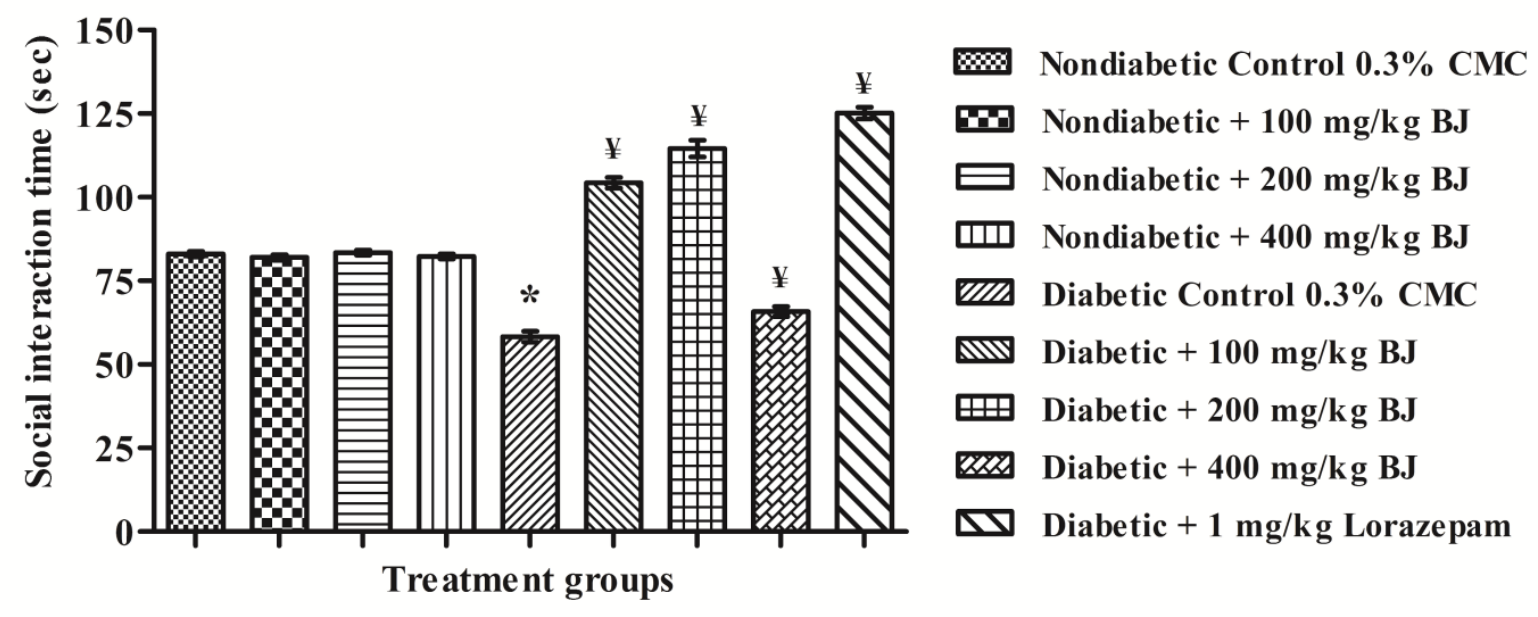

Fig. 3. Effect of Brassica juncea on social interaction behaviour in nondiabetic and diabetic rats. ${ }^{*} p<0.05$ vs. Nondiabetic Control; ${ }^{¥} p<0.05$ vs. Diabetic Control.

of action of Brassica juncea seeds have been made in an earlier independent report (Yadav et al., 2004).

Insulin dependent as well as insulin resistant diabetic patients are also prone to mental health problems, and exaggerated states of both anxiety and depression are also observed in rodent models of both types 1 and type 2 diabetes (Hutter et al., 2010; Regan, 2012). Alloxan-induced diabetes is a well known animal model for insulin dependent diabetes (Lenzen, 2008; Rohilla and Ali, 2012), which can also lead to insulin resistance (Reaven et al., 1977). Exaggerated behavioural symptoms of anxiety in alloxan-diabetic rats were clearly observed in all the three behavioural tests used in this study, and BJ treatments effectively antagonized all anxiety symptoms quantified in our experiments. These observations strongly suggest that exaggerated anxiety induced by hyperglycemia can be suppressed and eliminated by repeated daily oral doses of the extract. Since BJ treatments in nondiabetic animals did not have any significant effect on any anxiety-associated behavioural parameters quantified, the mechanism and processes involved in its observed anxiolytic-like effects in diabetic animals are most probably not analogous to those known for psychotherapeutics now commonly used for combating anxiety disorders. In general, the anxiolytic activities of lorazepam and other anxiolytics are observed as well in nondiabetic animals.

It must be noted that even after the lowest BJ dose tested (100 mg/kg/day), an almost complete reversal of all altered behavioural anxiety symptoms in diabetic rats was observed, and that such efficacies of the extract after its highest dose (400 $\mathrm{mg} / \mathrm{kg} / \mathrm{day}$ ) tested were always quantitatively lower than its lower ones. These observations suggest that the dose response relationship of BJ for its anxiolytic-like activities follows, most probably, an inverted U-shape curve. Many such effects of dietary phytochemicals are known (Son et al., 2008), and they can now be well explained by the hormesis paradigms for cellular stress responses (Calabrese et al., 2010). Such were not the cases for antihyperglycemic or antidepressants-like activities of $\mathrm{BJ}$ in the alloxan diabetic animals observed earlier in our laboratories (Unpublished data). Both these activities of the extract increased somewhat with its increasing doses, whereupon its maximum efficacies were observed after 200 $\mathrm{mg} / \mathrm{kg} /$ day dose regimen, and the efficacy of this dose regimen was almost equal to that of the highest one tested (400 $\mathrm{mg} / \mathrm{kg} / \mathrm{day})$. Thus, it seems reasonable to assume that biological processes and neurological mechanisms involved in antidepressant and anxiolytic-like effects in diabetic animals are not identical. The presence of functionally different types of Brassica juncea constituents in the extract could be a possible explanation for the observed difference between the shapes of the dose response curves for its antidepressant and anxiolytic activities. Comparative studies with diverse types of Brassica juncea extracts and their constituents, and more precise dose response experiments could be a feasible means for better clarification of the observed lowered efficacy of the highest BJ dose tested.

Our interest in testing the potential antidepressant and anxiolytic-like activities of Brassica juncea leaves was triggered initially by the reports revealing antidepressants and anxiolytics-like efficacies of two BJ constituents isorhamnetin and sinapic acid in nondiabetic animals (Chatterjee et al., 2005; Yoon et al., 2007). The presence of both these polyphenolics in BJ was confirmed by analysis. Therefore, an observed lack of antidepressant and anxiolytic activities of BJ in nondiabetic animals could indicate that either their concentrations in the extract are not high enough, or that some other constituents of the extract modulate their bioactivities. However, since both these activities of the extract were apparent in diabetic animals it could as well be that extractable Brassica juncea leaf constituents other than these two are the major central nervous system function modulating components of BJ. Clarification of the situation has to wait until the results of our still ongoing studies with different types of extracts containing varying concentrations of polyphenols are available. For such purposes, we have now identified a convenient pharmacological screening model which is currently being experimentally verified for its predictive validity. If verified, this rodent model could not only be useful for identifying the bioactive constituents of BJ, but also for psychopharmacological studies with other commonly consumed vegetables with higher contents of polyphenolic and other antioxidative compounds.

Observations reported in this communication constitute a part of a newly initiated project directed towards the identification of healthy vegetables and fruits potentially useful for combating the diabesity, i.e. the spreading epidemic of the $21^{\text {st }}$ century. Hereupon, a holistic pharmacological strategy based on the current concepts on psychobiological processes involved in health and diseases is used (Chatterjee and Kumar, 2012; Hamilton-West, 2011). This strategy evolved from our observations initially made with a few medicinal plants well known to the western herbalists (Kumar and Chatterjee, 2008). During the course of our more recent effort we have identified psychopharmacological activities of some well-known 
secondary plant metabolites with bacteriostatic activities. One of them is fumaric acid (Araujo et al., 2011), which is also known to occur in mustard (DeKock and Morrison, 1958). Encouraged by the observations made to date with BJ, attempts to test possible involvement of fumarate in the pharmacological activity profile of Brassica juncea leaves seem warrantable.

Although during more recent years numerous efforts have been made to demonstrate the health promoting properties of phytochemicals of Brassicaceae (Curciferae) family (Cartea et al., 2011; Jahangir et al., 2009), most of them have neglected the possibility that they could be useful for prevention of mental health problems commonly encountered in almost all chronically ill patients. Appropriate uses of conventionally known behavioural models using animals with pathologies could be a promising first step for better understanding the broad spectrum of health benefits potentially offered by diverse such and other vegetables and fruits commonly consumed in different countries. Observation made to date with BJ add further experimental evidences to this conviction, and strongly suggest Brassica juncea leaves could as well be an interesting starting point for obtaining nutraceuticals or phytotherapeutics potentially useful for combating diabesity associated psychopathologies. Since polyphenolics and other known bioactive components of Brassica juncea leaves are also encountered in numerous other vegetables and fruits, observations made during efforts to experimentally verify this possibility could as well be useful for identifying other edibles that could more rationally enrich the modern dietary recommendations.

Psychopharmacological studies with vegetables and fruits using animals with metabolic disorders could be a feasible means for identifying plants and fruits particularly suited for combating mental health problems commonly associated with such disorders. Brassica juncea leaves seem to be one such vegetable potentially useful for reducing the health care costs of diabetic patients suffering from comorbid anxiety disorders.

\section{ACKNOWLEDGEMENTS}

Authors would like to acknowledge technical support from Natural Remedies Pvt. Ltd., Bangalore, in analytical characterization of Brassica juncea extract. Ajit Kumar Thakur is thankful to the University Grants Commission, New Delhi, India for providing the financial assistance.

\section{CONFLICT OF INTERESTS}

The authors declare that there are no conflicts of interest in this study.

\section{REFERENCES}

Araujo WL, Nunes-Nesi A, Fernie AR. Fumarate: Multiple functions of a simple metabolite. Phytochemistry. 2011;72:838-843.

Balhara YPS. Diabetes and psychiatric disorders. Indian J Endocrinol Metab. 2011;15:274-283.

Bronstein PM. Repeated trials with the albino rat in the open field as a function of age and deprivation. J Comp Physiol Psychol. 1972;81:84-93.

Calabrese V, Cornelius C, Dinkova-Kostova AT, Calabrese EJ, TANG / www.e-tang.org
Mattson MP. Cellular stress responses, the hormesis paradigm, and vitagenes: novel targets for therapeutic intervention in neurodegenerative disorders. Antioxid Redox Signal. 2010;13:1763-1811.

Cartea ME, Fransisco M, Soengas P, Velasco P. Phenolic compounds in Brassica vegetables. Molecules. 2011;16:251-280.

Chandrasekaran CV, Thiyagarajan P, Sundarajan K, Goudar KS Deepak M. Evaluation of the genotoxic potential and acute oral toxicity of standardized extract of Andrographis paniculata (KalmColdTM). Food Chem Toxicol. 2009;47:1892-1902.

Chatterjee SS, Kumar V. Holistic Psychopharmacology and Promiscuous Plants and Principles of Ayurveda. Am J Plant Sci 2012;3:1015-1021.

Chatterjee SS, Noeldner M, Schoetz K. Use of rutin and isorhamnetin for treating depressive states and depression and other emotion disorders. Bioplanta Arzneimittel. 2005;EP1599211.

DeKock PC, Morrison RI. The metabolism of chlorotic leaves. 2. Organic acids. Biochem J. 1958;70:272-277.

Diamond J. Medicine: diabetes in India. Nature. 2011;469:478-479.

Faulkner GE, Gorczynski PF, Cohn TA. Psychiatric illness and obesity: recognizing the "obesogenic" nature of an inpatient psychiatric setting. Psychiatr Serv. 2009;60:538-541.

File SE, Hyde JR. Can social interaction be used to measure anxiety?. Br J Pharmacol. 1978;62:19-24.

Grover JK, Yadav S, Vats V. Hypoglycemic and antihyperglycemic effect of Brassica juncea diet and their effect on hepatic glycogen content and the key enzymes of carbohydrate metabolism. Mol Cell Biochem. 2002;241:95-101.

Hamilton-West K. Psychobiological processes in health and illness. (California, USA: SAGE Publication Inc.), 2011.

Herzer M, Hood KK. Anxiety symptoms in adolescents with type 1 diabetes: association with blood glucose monitoring and glycaemic control. J Pediatr Psychol. 2010;35:415-425.

Hutter N, Schnurr A, Baumeister H. Healthcare costs in patients with diabetes mellitus and comorbid mental disordersa systematic review. Diabetologia. 2010;53:2470-2479.

Jahangir M, Kim HK, Choi YH, Verpoorte R. Health-Affecting Compounds in Brassicaceae. Compr Rev Food Sci Food Safety. 2009;8:31-43.

Kumar V, Chatterjee SS. Ethnopharmacology and rational evaluation of herbal remedies. In Handbook of Ethnopharmacology, Eddouks M ed. $1^{\text {st }}$ ed. (Trivandrum, India: Research Signpost Publishers), pp. 25-50, 2008.

Kumar V, Thakur AK, Barothia ND, Chatterjee SS. Therapeutic potentials of Brassica juncea: an overview. TANG. 2011;1:e2.

Lenzen S. The mechanisms of alloxan- and streptozotocin-induced diabetes. Diabetologia. 


\section{8;51:216-226.}

Malgorzata NK, Aleksander S. Changes of phenolic content in rapeseed during preliminary drying. $J$ Oilseed Brassica. 2010;1:33-38.

Malvi R, Jain S, Khatri S, Patel A, Mishra S. A review on Antidiabetic medicinal plants and marketed herbal formulations. Int J Pharm Biol Arch. 2011;2:1344-1355.

Manohar PR, Pushpan R, Rohini S. Mustard and its uses in Ayurveda. Indian J Tradit Knowl. 2009;8:400-404

Mukherjee PK, Maiti K, Mukherjee K, Houghton PJ. Leads from Indian medicinal plants with hypoglycemic potentials. J Ethnopharmacol. 2006;106:1-28.

Pan A, Sun Q, Okereke OI, Rexrode KM, Rubin RR, Lucas M, Willett WC, Manson JE, Hu FB. Use of antidepressant medication and risk of type 2 diabetes: results from three cohorts of US adults. Diabetologia. 2012;55:63-72.

Pellow S, File SE. Anxiolytic and anxiogenic drug effects on exploratory activity in an elevated plus maze: a novel test of anxiety in the rat. Pharmacol Biochem Behav. 1986;24:525-529.

Pimentel GD, Micheletti TO, Pace F, Rosa JC, Santos RV, Lira FS. Gut-central nervous system axis is a target for nutritional therapies. Nutr J. 2012;11:22.

Rahmatullah M, Shefa TF, Hasan L, Hossain MT, Ahmed S, Mamun AA, Islam MR, Rahman S, Chowdhury MH. A study on antinociceptive and anti-hyperglycemic activity of methanol extract of Brassica juncea (L.) Czern. leaves in mice. Adv in Nat Appl Sci. 2010;4:221-225.

Reagan LP. Diabetes as a chronic metabolic stressor: causes, consequences and clinical complications. Exp Neurol. 2012;233:68-78.

Reaven GM, Sageman WS, Swenson RS. Development of insulin resistance in normal dogs following alloxan-induced insulin deficiency. Diabetologia. 1977;13:459-462.

Rohilla A, Ali S. Alloxan Induced Diabetes: Mechanisms and Effects. Int J Res Pharma Biomedical Sci. 2012;3:819-823.

Sekirov I, Russell SL, Antunes LC, Finlay BB. Gut microbiota in health and disease. Physiol Rev. 2010;90:859-904.

Son TG, Camandola S, Mattson MP. Hormetic dietary phytochemicals. Neuromolecular Med. 2008;10:236-246.

Valavala VK, Vangipurapu RK, Banam VR, Pulukurthi UMR, Turlapati NR. Effect of Mustard (Brassica juncea) leaf extract on Streptozotocin-induced Diabetic cataract in Wistar rats. J Food Biochem. 2011;35:109-124.

Yadav SP, Vats V, Ammini AC, Grover JK. Brassica juncea (Rai) significantly prevented the development of insulin resistance in rats fed fructose-enriched diet. J Ethnopharmacol. 2004;93:113-116.

Yoon BH, Jung JW, Lee JJ, Cho YW, Jang CG, Jin C, Oh TH, Ryu JH. Anxiolytic-like effects of sinapic acid in mice. Life Sci. 2007;81:234-240. 